\title{
Two-Player Location Game in a Closed-Loop Market with Quantity Competition
}

\author{
Xiaofeng Chen $\mathbb{D}^{1,2}$ Qiankun Song, ${ }^{2}$ and Zhenjiang Zhao ${ }^{3}$ \\ ${ }^{1}$ School of Economics and Management, Chongqing Jiaotong University, Chongqing 400074, China \\ ${ }^{2}$ Department of Mathematics, Chongqing Jiaotong University, Chongqing 400074, China \\ ${ }^{3}$ Department of Mathematics, Huzhou University, Huzhou 313000, China \\ Correspondence should be addressed to Xiaofeng Chen; xxffch@126.com
}

Received 7 July 2020; Revised 8 August 2020; Accepted 14 August 2020; Published 25 August 2020

Academic Editor: Sabri Arik

Copyright (c) 2020 Xiaofeng Chen et al. This is an open access article distributed under the Creative Commons Attribution License, which permits unrestricted use, distribution, and reproduction in any medium, provided the original work is properly cited.

This paper considers the two-player location game in a closed-loop market with quantity competition. Based on the Cournot and Hotelling models, a circle model is established for a closed-loop market in which two players (firms) play a location game under quantity competition. Using a two-stage (location-then-quantity) pattern and backward induction method, the existence of subgame-perfect Nash equilibria is proved for the location game in the circle model with a minimum distance transportation cost function. In addition, sales strategies are proposed for the two players for every local market on the circle when the players are in the equilibrium positions. Finally, an algorithm for simulating the competitive dynamics of the closed-loop market is designed, and two numerical simulations are provided to substantiate the effectiveness of the obtained results.

\section{Introduction}

Game theory, the science of strategy, was pioneered by John von Neumann when he proved the basic principles in 1928 [1]. As one of the main basic analysis tools for phenomena related to struggle or competition, game theory has been widely applied in politics, international relations, military strategy, biology, economics, computer science, and many other fields [2-7].

The location problem is an important topic in the fields of supply chain management and industrial organization. In 1929, Hotelling first introduced the game theory to the location problem and established the classic Hotelling model [8]. In this model, consumers are assumed to be evenly distributed on a linear street, and two companies of the same size that produce homogeneous goods determine their locations such that the profits are maximized. During the following decades, researchers investigated variations of the location problem based on the classic model and obtained a variety of results. In [9], the authors claimed that a price equilibrium solution exists everywhere in a modified version of the Hotelling model and showed that both sellers tend to maximize their differentiation for this model. In [10], the authors studied Cournot competition in linear city model with a nonuniform consumer distribution and derived a necessary condition for an agglomeration equilibrium. In [11], the authors proved that a mixed strategy equilibrium exists for the Hotelling model if a pure strategy equilibrium does not exist. In [12], the authors extended the Hotelling spatial competition model in three aspects, namely, the number of firms, shape of the demand curve, and type of space. In [13], the author examined the Hotelling model for duopolistic competition with a class of utility functions and proved the existence of an equilibrium when the curvature of the utility functions is sufficiently high. In [14], the authors analyzed the relationship between consumer density and the equilibrium locations of the Hotelling model and noted that the equilibrium locations are closer if the density is higher. In [15], the author considered the problem of the existence of equilibrium states in the Hotelling model in the $n$-player case and analyzed the influence of the number of firms on the equilibrium outcome of Hotelling games. In 
[16], the authors discussed the Hotelling duopoly model with network effects and brand loyalty and showed that a pure strategy price equilibrium exists if the transportation costs are linear functions. In [17], the authors considered the influence of production technology and labor inputs on spatial competition in Hotelling model and found that the production technology is the main influence on the equilibrium locations. It was proved that a pure strategy price-location Nash equilibrium exists in the Hotelling duopoly model under general conditions on the cost-of-location function in [18]. In [19], the authors developed a duopoly game using the Hotelling model to research the competition between brick-and-mortar retailers and online retailers.

In general, the real market cannot be described by a linear segment with evenly distributed customers such as Hotelling model and its extended versions. In actual situations, markets are distributed along complex transportation networks. Therefore, many researchers considered spatial competition in the pattern of circles and complex networks to more accurately represent actual markets. For example, the existence of an equilibrium in the circle model was proved by using a two-stage (location-then-price) method in [20]. It was shown that a unique price equilibrium exists on a circular road when the transportation cost function is quadratic in [21]. In [22], the authors studied the location game in a circular market and demonstrated that the equidistant location pattern is the unique equilibrium location for the players in this market. In [23], the authors investigated two shipping duopoly models in circular markets and proved the existence and nonexistence of the equilibrium in different models. In [24], the authors considered spatial competition among for a multiplant Cournot oligopoly in a circular city and proved the uniqueness of the equilibrium location if the number of plants is equal for the two firms. In [25], the authors derived some conditions for even spacing to be an equilibrium of a circular market based on a two-stage approach. In [26], the authors investigated spatial Cournot competition in a circular city and showed that nonmaximum dispersion is the unique location equilibrium when duopoly firms deliver products in different transportation modes.

Strongly motivated by the above discussion, in this paper, we investigate the two-player location game in a closed-loop market. In such a game, two players develop quantity competition in the market, whose shape can be viewed as a circle. The goal of each player is to choose the optimal point on the circle as its location such that its profit is maximized. The main contributions of this paper are highlighted as follows:

(1) Not only is the optimal equilibrium state of the location game investigated, but also the optimal strategy, including the price and quantity plan for each player, is proposed.

(2) A computer algorithm is designed to simulate the evolutionary process of the two-player game in a closed-loop market. The simulation examples demonstrate the effectiveness and feasibility of the algorithm.
(3) It is shown that the two players follow the principle of maximum differentiation to choose their optimal locations in a closed-loop market model, which is strikingly different from Hotelling's minimum differentiation principle for a linear market model.

The remainder of this paper is organized as follows. In Section 2, some descriptions and assumptions for the twoplayer location game in a closed-loop market are introduced, and some important lemmas are provided. In Section 3, the two-player quantity competition in the closed-loop market is considered when the players' locations are fixed. The twoplayer location game in a closed-loop market is discussed in Section 4. An algorithm for simulating the dynamic evolution of the two players in the market is presented in Section 5. Finally, some conclusions are drawn in Section 6.

To end this section, we give some notations used in this paper. $x \in[0,1)$ stands for the position of the local market in a closed-loop market. $x_{1}$ and $x_{2}$ represent the positions of firms $A$ and $B$, respectively. $p$ is the price of the product, while $q_{1}$ and $q_{2}$ are the quantities produced by the two players. The function $c_{i}$ represents the transportation cost per unit product from the position $x_{i}$ to $x$, where $i=1,2$.

\section{Basic Assumptions of the Closed- Loop Market}

Consider two firms $A$ and $B$ in a closed-loop market. Without loss of generality, it is assumed that the closed loop is a circle with a circumference of 1 . The points on the circle are arranged in the counterclockwise direction, and each point is called a local market. Consumers are evenly distributed on the circle with a density of 1 . Firms $A$ and $B$ are distributed at two points on the circle (as shown in Figure 1(a)), and each firm produces homogeneous products with zero production cost.

For convenience of representation, the circle is disconnected at a certain point $O$ in Figure 1(a), and the circle is straightened into a line segment $O O^{\prime}$ of length 1 (as shown in Figure 1(b)). Clearly, the line $O O^{\prime}$ in Figure 1(b) can be transformed into the circle in Figure 1(a). Therefore, the circle is equivalent to the segment $O O^{\prime}$. Note that the two ends $O$ and $O^{\prime}$ of the line segment correspond to the same point $O$ on the circle. Take the left end $O$ of line $O O^{\prime}$ as the origin, and take the $O O^{\prime}$ direction as the positive direction to establish a number axis. Then, the coordinate value $x$ of a point on the right-half number axis represents the distance from the point to the origin $O$.

Suppose the locations of the firms $A$ and $B$ are, respectively, $x_{1}$ and $x_{2}$, where $x_{1}, x_{2} \in[0,1)$. The firms are responsible for the distribution of goods to the local market $x$, and the transportation cost is 1 per unit product per unit distance. There are two ways to transport products from location $x_{i}$ to the local market $x$, that is, counterclockwise and clockwise. We should choose the minimum distance for distribution. Therefore, the transportation cost per unit product from location $x_{i}$ to the local market $x$ is

$$
c_{i}(x)=\min \left\{\left|x_{i}-x\right|, 1-\left|x_{i}-x\right|\right\}, \quad i=1,2 .
$$




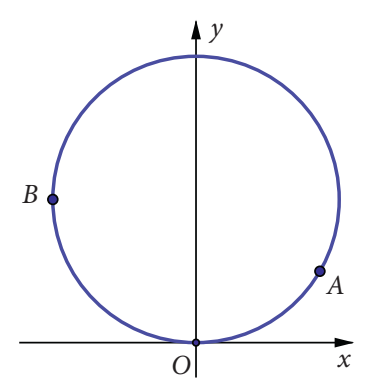

(a)

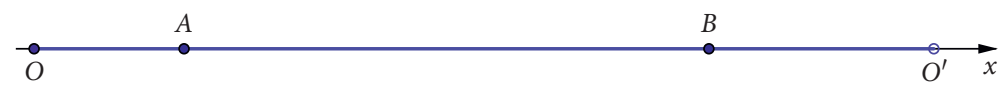

(b)

FIGURE 1: Circular market and its linearization. (a) Diagram of the circular market. (b) Linearization of the circle.

Clearly, $\quad c_{1}(x)=c_{2}(x) \quad$ when $\quad x_{1}=x_{2}, \quad$ and $c_{1}(x)+c_{2}(x)=1 / 2$ when $\left|x_{1}-x_{2}\right|=1 / 2$. The transportation costs of the two firms from the same location to the local market $x$ are equal if they are located at the same point, while the total transportation costs are $1 / 2$ if they are located at opposite ends of a certain diameter in the circle.

In the following sections, the total costs of transportation on the circle and the total profit of the firms, which are related to some integrals of $c_{i}(x)$, will be calculated. Therefore, we present some conclusions on integrals of $c_{i}(x)$.

Lemma 1. For $c_{i}(x)$ and $c_{i}^{2}(x)$, the following integrals hold:

$$
\begin{aligned}
& \int_{0}^{1} c_{i}(x) \mathrm{d} x=\frac{1}{4}, \\
& \int_{0}^{1} c_{i}^{2}(x) \mathrm{d} x=\frac{1}{12} .
\end{aligned}
$$

Lemma 2. For the integral of the product of $c_{1}(x)$ and $c_{2}(x)$, the following results are correct.

(i) When $\left(x_{1}, x_{2}\right) \in[0,(1 / 2)) \times[0,(1 / 2))$,

$$
\int_{0}^{1} c_{1}(x) c_{2}(x) \mathrm{d} x=\frac{2}{3}\left|x_{1}-x_{2}\right|^{3}-\frac{1}{2}\left(x_{1}-x_{2}\right)^{2}+\frac{1}{12} .
$$

(ii) When $\left(x_{1}, x_{2}\right) \in[0,(1 / 2)) \times[(1 / 2), 1)$,

$$
\int_{0}^{1} c_{1}(x) c_{2}(x) \mathrm{d} x=-\frac{2}{3}\left|x_{1}-x_{2}+\frac{1}{2}\right|^{3}+\frac{1}{2}\left(x_{1}-x_{2}+\frac{1}{2}\right)^{2}+\frac{1}{24} \text {. }
$$

(iii) When $\left(x_{1}, x_{2}\right) \in[(1 / 2), 1) \times[0,(1 / 2))$,

$$
\int_{0}^{1} c_{1}(x) c_{2}(x) \mathrm{d} x=-\frac{2}{3}\left|x_{2}-x_{1}+\frac{1}{2}\right|^{3}+\frac{1}{2}\left(x_{2}-x_{1}+\frac{1}{2}\right)^{2}+\frac{1}{24} \text {. }
$$

(iv) When $\left(x_{1}, x_{2}\right) \in[(1 / 2), 1) \times[(1 / 2), 1)$, $\int_{0}^{1} c_{1}(x) c_{2}(x) \mathrm{d} x=\frac{2}{3}\left|x_{1}-x_{2}\right|^{3}-\frac{1}{2}\left(x_{1}-x_{2}\right)^{2}+\frac{1}{12}$.

Specifically,

$$
\begin{aligned}
& \int_{0}^{1} c_{1}(x) c_{2}(x) \mathrm{d} x=\frac{1}{12}, \quad \text { if } x_{1}=x_{2}, \\
& \int_{0}^{1} c_{1}(x) c_{2}(x) \mathrm{d} x=\frac{1}{24}, \quad \text { if }\left|x_{1}-x_{2}\right|=\frac{1}{2} .
\end{aligned}
$$

The proofs of Lemmas 1 and 2 are presented in the appendix.

\section{Quantity Competition in the Closed- Loop Market}

In this section, we first recall the Cournot duopoly model proposed by Cournot in 1838 [27]. In this model, two players (firms) producing the same products compete by choosing their outputs independently under the assumption that their competitor does not change their output in response, which leads to a balanced result between competition and monopoly.

The basic assumptions of the Cournot model are that the market includes only two firms $A$ and $B$ that sell identical products; the production cost is zero (such as the acquisition of mineral water); the inverse demand function of the market is linear; firms $A$ and $B$ know the demand function of the market accurately; and both firms know each other's output. In this situation, each firm determines the output that can yield the maximum profit for them, which means that each firm passively adapts its output to the determined output of the other party. Next, we use the response function method to analyze the equilibrium state of production in the Cournot model.

Let the total market capacity be 1 and the inverse demand function be

$$
p=f\left(q_{1}+q_{2}\right)=1-q_{1}-q_{2},
$$

where $p$ is the price of the product and $q_{1}$ and $q_{2}$ are the quantities produced by firms $A$ and $B$, respectively. Then, the profits of firms $A$ and $B$ are as follows: 


$$
\begin{aligned}
& \pi_{1}\left(q_{1}, q_{2}\right)=p q_{1}=q_{1}-q_{1}^{2}-q_{1} q_{2}, \\
& \pi_{2}\left(q_{1}, q_{2}\right)=p q_{2}=q_{2}-q_{2}^{2}-q_{1} q_{2} .
\end{aligned}
$$

For firm $A$, the first-order condition maximizing its profit is that

$$
\begin{aligned}
& \frac{\partial \pi_{1}}{\partial q_{1}}=1-2 q_{1}-q_{2}=0, \\
& \text { or } q_{1}=\frac{1}{2}\left(1-q_{2}\right) .
\end{aligned}
$$

Equation (10) is firm $A$ 's best response function, which represents the optimal output of firm $A$ depending on the output of firm $B$. In other words, for each output of firm $B$, firm $A$ will respond and determine the product that can maximize its profit.

Similarly, for firm $B$, the first-order profit-maximizing condition is

$$
\begin{aligned}
& \frac{\partial \pi_{2}}{\partial q_{2}}=1-2 q_{2}-q_{1}=0, \\
& \text { or } q_{2}=\frac{1}{2}\left(1-q_{1}\right) .
\end{aligned}
$$

Equation (11) is firm B's best response function, which represents the relationship between the optimal output of firm B and the output of firm A.

By combining the best response functions (10) and (11), the equilibrium output solution of firms $A$ and $B$ can be obtained as follows:

$$
q_{1}^{*}=q_{2}^{*}=\frac{1}{3} .
$$

The equilibrium output of each firm is $1 / 3$ of the market capacity. As shown in Figure 2, the response functions of firms $A$ and $B$ are linear since the demand function is linear. The intersection $E$ of the two lines is the equilibrium solution of the Cournot model.

In the following, we consider the two-player game in a closed-loop market with quantity competition. Like demand function (8) in the Cournot model, for the closed-loop market, we assume that the demand function of the local market at $x$ is

$$
p(x)=1-q_{1}(x)-q_{2}(x), \quad \forall x \in[0,1),
$$

where $p(x)$ is the price of the product for the local market $x$ and $q_{1}(x)$ and $q_{2}(x)$ are the sales of firms $A$ and $B$ for the local market $x$, respectively. Therefore, the profits $\pi_{1}$ and $\pi_{2}$ of firms $A$ and $B$ in the local market $x$ are, respectively,

$$
\begin{aligned}
& \pi_{1}=\left[1-q_{1}(x)-q_{2}(x)-c_{1}(x)\right] q_{1}(x), \\
& \pi_{2}=\left[1-q_{1}(x)-q_{2}(x)-c_{2}(x)\right] q_{2}(x),
\end{aligned}
$$

where $c_{i}(x)$, defined in (1), represents the transportation cost per unit product from location $x_{i}$ of the firm to the local market $x$. In a similar way to the response function approach for analyzing the Cournot model, we can extend the discussion. By calculating the partial derivatives of (14) and

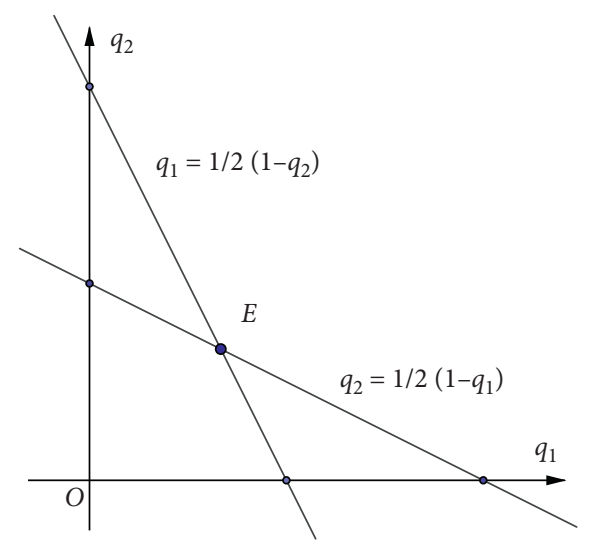

FIgUre 2: The response functions in the Cournot model.

(15), the first-order conditions for maximizing the profits of firms $A$ and $B$ in the local market $x$ are obtained as follows:

$$
\left\{\begin{array}{l}
\frac{\partial \pi_{1}}{\partial q_{1}}=1-2 q_{1}(x)-q_{2}(x)-c_{1}(x)=0, \\
\frac{\partial \pi_{2}}{\partial q_{2}}=1-q_{1}(x)-2 q_{2}(x)-c_{2}(x)=0 .
\end{array}\right.
$$

Then, the response functions of firms $A$ and $B$ in local market $x$ are as follows:

$$
\begin{aligned}
& q_{1}(x)=\frac{1}{2}\left[1-q_{2}(x)-c_{1}(x)\right], \\
& q_{2}(x)=\frac{1}{2}\left[1-q_{1}(x)-c_{2}(x)\right] .
\end{aligned}
$$

According to the above equations, in the equilibrium state of output competition, the quantities of products sold by the two firms in local market $x$ are

$$
\begin{aligned}
& q_{1}^{*}(x)=\frac{1}{3}\left[1-2 c_{1}(x)+c_{2}(x)\right], \\
& q_{2}^{*}(x)=\frac{1}{3}\left[1+c_{1}(x)-2 c_{2}(x)\right] .
\end{aligned}
$$

Meanwhile, according to (14), (15), and (19), the profits of firms $A$ and $B$ in local market $x$ are

$$
\begin{aligned}
& \pi_{1}^{*}(x)=\frac{1}{9}\left[1-2 c_{1}(x)+c_{2}(x)\right]^{2}, \\
& \pi_{2}^{*}(x)=\frac{1}{9}\left[1+c_{1}(x)-2 c_{2}(x)\right]^{2} .
\end{aligned}
$$

By integrating (19) and employing Lemma 1, it can be obtained that the total outputs $Q_{1}^{*}$ and $Q_{2}^{*}$ of firms $A$ and $B$ are, respectively,

$$
\begin{aligned}
& Q_{1}^{*}=\int_{0}^{1} q_{1}^{*}(x) \mathrm{d} x=\frac{1}{4}, \\
& Q_{2}^{*}=\int_{0}^{1} q_{2}^{*}(x) \mathrm{d} x=\frac{1}{4},
\end{aligned}
$$


which means that the equilibrium output of each firm is $1 / 4$ of the market capacity.

Based on the above analysis, we can summarize the results in the following proposition.

Proposition 1. In a closed-loop market with inverse demand function (8) and transportation cost function (1), if two players compete with the quantity of outputs, their equilibrium outputs are both $1 / 4$, regardless of their locations $x_{1}$ and $x_{2}$.

Remark 1. Comparing the demand function (8) in the Cournot model with the demand function (13) in the closedloop market discussed in this section, we find that the meaning of (13) is the same as that of (8) if $x$ in (13) is fixed. Therefore, the output competition in each local market $x$ on the circle can be regarded as a Cournot model with quantity competition, while the whole circle market can be regarded as being composed of an infinite number of Cournot models.

Remark 2. Comparing the response functions (10) and (11) in the Cournot model with the response functions (17) and (18) in the closed-loop market model, we find that the outputs $q_{i}(x)$ in response functions (17) and (18) must deduce quantities whose values are equivalent to the transportation cost in the closed-loop market. As a result, the proportion of the equilibrium output of each firm to the total market capacity is reduced by $1 / 12$.

\section{Location Game in the Closed-Loop Market}

In the previous section, we assumed that positions $x_{1}$ and $x_{2}$ of firms $A$ and $B$ are fixed. In this section, we consider the locations of the firms as decision variables. Therefore, this section considers how firms $A$ and $B$ choose their optimal locations $x_{1}$ and $x_{2}$ to obtain the maximum profits through quantity competition.

In this situation, we can analyze the problem as a complete information dynamic game process composed of two stages. In the first stage of the game, the two firms choose their locations in the circle market independently. Then, in the second stage, each firm launches the output competition after observing the location of the other firm. We adopt backward induction to solve the subgame-perfect Nash equilibria of this game.

In the second stage, each firm observes the position of the other firm and then competes for outputs. Therefore, the analysis approach in this stage is the same as that in the previous section. Since we still assume that the demand function $p(x)$ is in the form of (13), the product quantities $q_{1}^{*}(x)$ and $q_{2}^{*}(x)$ sold by firms $A$ and $B$ in the local market $x$ are still in the form of (19) when the output competition reaches the equilibrium state. Therefore, the profits $\pi_{1}^{*}$ and $\pi_{2}^{*}$ of firms $A$ and $B$ are still calculated according to (20) in the local market $x$.

In the first stage, each firm chooses its own optimal location to maximize its profit. The total profits $\Pi_{i}$ of firms $A$ and $B$ in the whole closed-loop market are

$$
\Pi_{i}\left(x_{1}, x_{2}\right)=\int_{0}^{1} \pi_{i}^{*} \mathrm{~d} x, \quad i=1,2 .
$$

Substituting (20) into (22) and according to Lemma 1, it can be seen that

$$
\Pi_{i}\left(x_{1}, x_{2}\right)=\frac{11}{108}-\frac{4}{9} \int_{0}^{1} c_{1}(x) c_{2}(x) \mathrm{d} x .
$$

Then, the first-order conditions of profit maximization are that

$$
\begin{cases}\frac{\partial \Pi_{1}}{\partial q_{1}}=0, & \text { or } \frac{\partial \Pi_{1}}{\partial q_{1}} \text { does not exist } \\ \frac{\partial \Pi_{2}}{\partial q_{2}}=0, & \text { or } \frac{\partial \Pi_{2}}{\partial q_{2}} \text { does not exist }\end{cases}
$$

which are equivalent to

$$
\begin{cases}\frac{\partial}{\partial x_{1}} \int_{0}^{1} c_{1}(x) c_{2}(x) \mathrm{d} x=0, & \text { or } \frac{\partial}{\partial x_{1}} \int_{0}^{1} c_{1}(x) c_{2}(x) \mathrm{d} x \text { does not exist } \\ \frac{\partial}{\partial x_{2}} \int_{0}^{1} c_{1}(x) c_{2}(x) \mathrm{d} x=0, & \text { or } \frac{\partial}{\partial x_{2}} \int_{0}^{1} c_{1}(x) c_{2}(x) \mathrm{d} x \text { does not exist. }\end{cases}
$$

We discuss $\left(x_{1}, x_{2}\right)$ and whether they satisfy conditions (25) in four cases: (i) $\left(x_{1}, x_{2}\right) \in[0,(1 / 2)) \times[0,(1 / 2))$, (ii) $\left(x_{1}, x_{2}\right) \in[0,(1 / 2)) \times[(1 / 2), 1)$, (iii) $\left(x_{1}, x_{2}\right) \in[(1 / 2), 1) \times$ $[0,(1 / 2))$, and (iv) $\left(x_{1}, x_{2}\right) \in[(1 / 2), 1) \times[(1 / 2), 1)$.

For cases (i) and (iv), $\partial / \partial x_{1} \int_{0}^{1} c_{1}(x) c_{2}(x) \mathrm{d} x$ does not exist if $x_{1}=x_{2}$ according to Lemma 2. Hence, conditions (25) are satisfied if $x_{1}=x_{2}$. Similarly, for case (ii), conditions (25) are satisfied if $x_{2}-x_{1}=1 / 2$. For case (iii), conditions (25) are satisfied if $x_{1}-x_{2}=1 / 2$. Therefore, the possible equilibrium positions of the two firms are at the same point or at opposite ends of a diameter of the circular market.

According to (23) and Lemma 2, we can calculate the profits of the two firms in the possible equilibrium locations as follows: 


$$
\Pi_{i}= \begin{cases}\frac{7}{108}, & x_{1}^{*}=x_{2}^{*} \text { (at the same point), } \\ \frac{1}{12}, & \left|x_{1}^{*}-x_{2}^{*}\right|=\frac{1}{2},(\text { at opposite ends of a diameter). }\end{cases}
$$

This result indicates that the maximum profit of the two firms at opposite ends of a diameter is larger than that at the same point. Next, we analyze the sales program, including sales volume and sales price, for the firms in the two possible equilibrium locations.

If the equilibrium location is at the same point, that is, $x_{1}^{*}=x_{2}^{*}$, according to (19) and (13), noting that $c_{1}(x)=c_{2}(x)$, each firm chooses a sales quantity $q_{i}^{*}(x)$ and a corresponding sales price $p^{*}(x)$ for the local market $x$ as follows:

$$
\begin{aligned}
& q_{1}^{*}(x)=q_{2}^{*}(x)=\frac{1}{3}\left[1-c_{i}(x)\right], \\
& p^{*}(x)=\frac{1}{3}\left[1+2 c_{i}(x)\right] .
\end{aligned}
$$

Then, the total sales quantity $Q_{i}^{*}$ of each firm in the whole market is

$$
Q_{1}^{*}=Q_{2}^{*}=\int_{0}^{1} q_{i}^{*}(x) \mathrm{d} x=\frac{1}{4} .
$$

In this situation, as shown in Figure 3, the sales price $p^{*}(x)$ of the firms is positively correlated with the transportation $\operatorname{cost} c_{i}(x)$ and negatively correlated with the sales volume $q_{i}^{*}(x)$. Therefore, the price increases with an increase in the cost, while the sales volume decreases with an increase in the price.

If the equilibrium location is at opposite ends of a diameter, that is, $\left|x_{1}^{*}-x_{2}^{*}\right|=1 / 2$, according to (19) and noting that $c_{1}(x)+c_{2}(x)=1 / 2$, each manufacturer chooses a sales quantity $q_{i}^{*}(x)$ and a corresponding sales price $p^{*}(x)$ for the local market $x$ as follows:

$$
\begin{aligned}
& q_{1}^{*}(x)=c_{2}(x), \\
& q_{2}^{*}(x)=c_{1}(x), \\
& p^{*}(x)=1-c_{1}(x)-c_{2}(x)=\frac{1}{2} .
\end{aligned}
$$

In this situation, as shown in Figure 4, the sales price $p^{*}(x)$ of the two firms is a constant, while the sales volume $q_{i}^{*}(x)$ is negatively related to the transportation cost $c_{i}(x)$. Thus, regardless of how the cost and sales volume change, the price is always constant, and the sales volume decreases as the cost increases.

Summarizing the above discussion, we obtain the conclusions shown in Table 1.

In fact, strategy 1 is not optimal. Based on (23) and Lemma 2, the second-order conditions can be calculated as

$$
\lim _{x_{1} \longrightarrow x_{2}} \frac{\partial^{2} \Pi_{i}}{\partial x_{i}^{2}}=\frac{4}{9}>0
$$

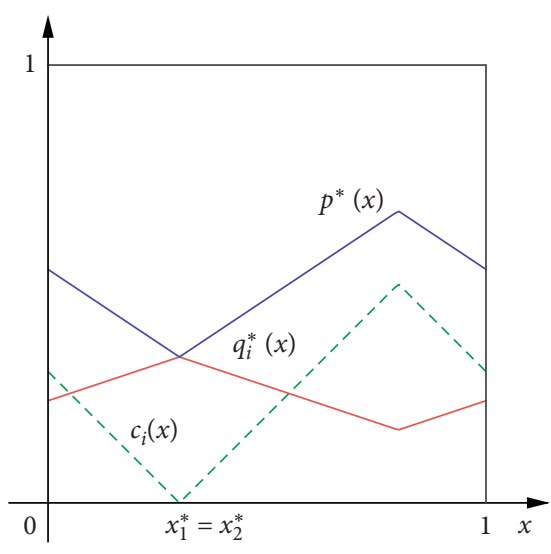

Figure 3: Price and quantity curves with the equilibrium positions at the same point.

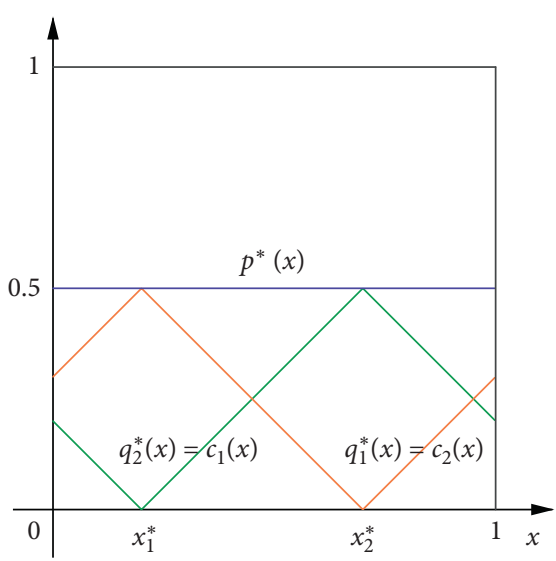

Figure 4: Price and quantity curves with equilibrium positions at opposite ends of a diameter.

Therefore, the second-order condition of maximizing $\Pi_{i}\left(x_{1}, x_{2}\right)$ is not satisfied if $x_{1}=x_{2}$. In addition, the profit $\Pi_{i}\left(x_{1}, x_{2}\right)$ can also be Pareto improved. For example, the profit $\Pi_{i}\left(x_{1}, x_{2}\right)$ of each firm is $7 / 108$ if the firms are located at the same point; that is, $x_{1}=x_{2}$. Now, assume that firm $A$ 's position is fixed at $x_{1}$ and firm $B$ 's position $x_{2}$ is changed slightly, that is, $x_{2}=x_{1}+\delta$, where $\delta$ is a number close to but not zero. According to (23) and Lemma 2, the increment $\Delta \Pi_{i}$ of the profit can be calculated as follows:

$$
\Delta \Pi_{i}=\Pi_{i}\left(x_{1}, x_{1}+\delta\right)-\Pi_{i}\left(x_{1}, x_{2}\right)=\frac{2}{9} \delta^{2}-\frac{8}{27}|\delta|^{3}>0,
$$

which shows that the profits of firms $A$ and $B$ have both increased.

Strategy 2 can be viewed as optimal because it cannot be Pareto improved. If the positions of firms $A$ and $B$ are changed from $\left|x_{1}-x_{2}\right|-(1 / 2)=0$ to $\left|x_{1}-x_{2}\right|-(1 / 2)=\delta$, where $\delta$ represents a number close to but not equal to zero, then the increment $\Delta \Pi_{i}$ of the profit can be calculated as

$$
\Delta \Pi_{i}=\frac{8}{27}|\delta|^{3}-\frac{2}{9} \delta^{2}<0 .
$$


TABLE 1: Location game strategies based on possible equilibrium positions.

\begin{tabular}{|c|c|c|c|c|c|c|}
\hline \multirow{2}{*}{ Strategy } & \multirow{2}{*}{ Equilibrium points } & \multirow{2}{*}{ Cost } & \multicolumn{3}{|c|}{ Sales program } & \multirow{2}{*}{ Profit } \\
\hline & & & Price & Quantity & Total quantity & \\
\hline 1 & $x_{1}^{*}=x_{2}^{*}$ & $c_{1}(x)=c_{2}(x)$ & $(1 / 3)\left[1+2 c_{i}(x)\right]$ & $(1 / 3)\left[1-c_{i}(x)\right]$ & $Q_{1}^{*}=Q_{2}^{*}=(1 / 4)$ & $7 / 108$ \\
\hline 2 & $\left|x_{1}^{*}-x_{2}^{*}\right|=(1 / 2)$ & $c_{1}(x)+c_{2}(x)=(1 / 2)$ & $1 / 2$ & $\begin{array}{l}q_{1}^{*}(x)=c_{2}(x) \\
q_{2}^{*}(x)=c_{1}(x)\end{array}$ & $Q_{1}^{*}=Q_{2}^{*}=(1 / 4)$ & $1 / 12$ \\
\hline
\end{tabular}

Thus, the profit of each firm decreases.

Based on the above discussion, we summarize what we have demonstrated.

Proposition 2. In the closed-loop market with inverse demand function (8) and transportation cost function (1), if the two players develop the location game with quantity competition, the equilibrium locations $x_{1}^{*}$ and $x_{2}^{*}$ satisfy $\left|x_{1}^{*}-x_{2}^{*}\right|=(1 / 2)$, which means that they are at opposite ends of a diameter of the market. Furthermore, when the firms are at their equilibrium locations, the sales strategies, including price and quantity, in the local market $x$ are as follows:

$$
\begin{aligned}
& p^{*}(x)=\frac{1}{2}, \\
& q_{1}^{*}(x)=\min \left\{\left|x_{2}^{*}-x\right|, 1-\left|x_{2}^{*}-x\right|\right\}, \\
& q_{2}^{*}(x)=\min \left\{\left|x_{1}^{*}-x\right|, 1-\left|x_{1}^{*}-x\right|\right\} .
\end{aligned}
$$

Remark 3. Proposition 2 indicates that when the two players are at the equilibrium locations, they should provide different quantities of products for different local markets. In the quantity strategy, a player provides greater quantities to the local market that is closest to its location. However, the player adopts the same price strategy for every local market, which means that the price is indiscriminately unified in the entire closed-loop market.

Remark 4. For a linear city model, H. Hotelling proposed the principle of minimum differentiation, according to which, two competing players will be located in the same location [8]. However, Proposition 2 describes the principle of maximum differentiation in this paper. In a closed-loop market, due to the nonlinearity of the transportation cost function (1), two players will keep the maximum dispersion between their locations, which is contrary to Hotelling's principle.

\section{Numerical Simulations}

To illustrate the competitive dynamics in the close-loop market and verify the validity of the results, we employ MATLAB to simulate the game process of players 1 and 2 (firms $A$ and $B$ ). At the beginning of the simulation, each player randomly and independently chooses a point $x_{i}$ on the circle as its position. Then, the two players start the first round of position adjustments based on their profits. Player 1 uses (23) to calculate its own profit $\Pi_{1}\left(x_{1}, x_{2}\right)$ based on its position $x_{1}$ and player 2's position $x_{2}$ and moves a small step around the circle in the appropriate direction such that its new position increases its profit. Then, player 2 computes its own profit $\Pi_{2}\left(x_{1}, x_{2}\right)$ and moves a small step around the circle in the same manner as player 1 . In this way, the two players adjust their positions in each round and move alternately around the circle until their profits no longer increase.

Therefore, the algorithm for simulating competitive dynamics in the close-loop market can be designed as follows.

Step 1: initialize the move step size $h$ with a small number. Initialize the positions $x_{1}$ and $x_{2}$ of the two players with two random numbers in $[0,1]$.

Step 2: use (23) to compute $M_{1}=\Pi_{1}\left(x_{1}, x_{2}\right)$, $M_{2}=\Pi_{1}\left(x_{1}+h, x_{2}\right)$, and $M_{3}=\Pi_{1}\left(x_{1}-h, x_{2}\right)$.

Step 3: if $M_{1}<M_{2}$, assign the value $x_{1}+h$ to $x_{1}$. If $M_{1}<M_{3}$, assign the value $x_{1}-h$ to $x_{1}$.

Step 4: use (23) to compute $N_{1}=\Pi_{2}\left(x_{1}, x_{2}\right)$, $N_{2}=\Pi_{2}\left(x_{1}, x_{2}+h\right)$, and $N_{3}=\Pi_{2}\left(x_{1}, x_{2}-h\right)$.

Step 5: if $N_{1}<N_{2}$, assign the value $x_{2}+h$ to $x_{2}$ and return to Step 2. If $N_{1}<N_{3}$, assign the value $x_{2}-h$ to $x_{2}$ and return to Step 2 .

Based on this algorithm, two numerical simulations are performed for different pairs of initial positions, and the dynamic evolution of the players in the closed-loop market is shown in Figures 5 and 6 . The initial positions of the two players are $x_{1}=0.06$ and $x_{2}=0.68$ for the first simulation in Figure 5 and $x_{1}=0.66$ and $x_{2}=0.52$ for the second simulation in Figure 6 . The number of rounds from the initial state to the equilibrium state is 6 in the first simulation and 18 in the second simulation since the distance between the two positions in the initial state is shorter in the first simulation. It is seen that in the last round the positions $x_{1}$ and $x_{2}$ are not changed and satisfy $\left|x_{1}-x_{2}\right|=(1 / 2)$ in each simulation.

Remark 5. In [26], the authors investigated the location game in a circular market and derived some conditions for guaranteeing the existence and uniqueness of the location equilibrium. Compared with this previous work, we have not only shown that a maximum dispersion is the location equilibrium in a closed-loop market, but also proposed the sales strategies for the two players in the equilibrium position. Furthermore, an algorithm is designed for simulating the competitive dynamics of a closed-loop market. To the best of our knowledge, this algorithm has not been proposed in the literature on circular markets. 


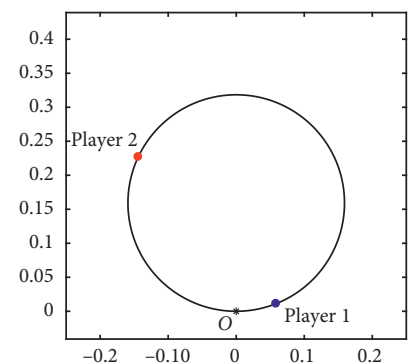

$\begin{aligned}-x_{1} & =0.06 \\ -x_{2} & =0.68\end{aligned}$

(a)

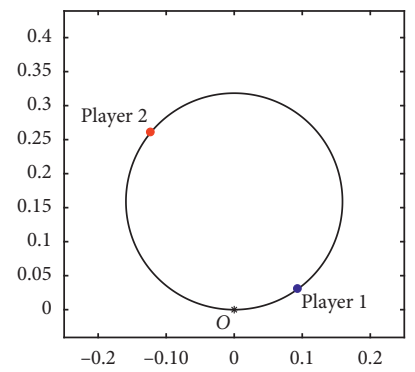

- $x_{1}=0.1$

- $x_{2}=0.64$

(e)

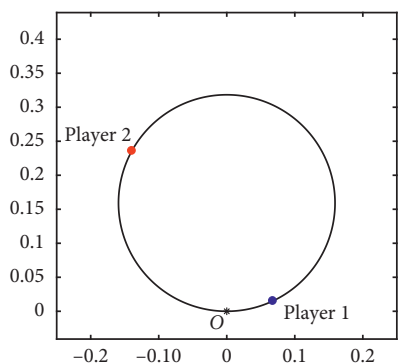

$-x_{1}=0.07$
$-x_{2}=0.67$

(b)

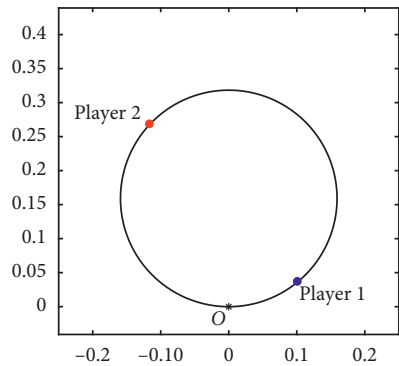

- $x_{1}=0.11$

- $x_{2}=0.63$

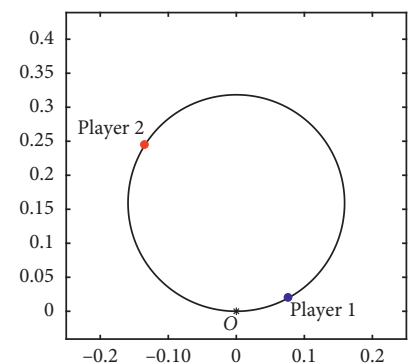

- $x_{1}=0.08$

- $x_{2}=0.66$

(c)

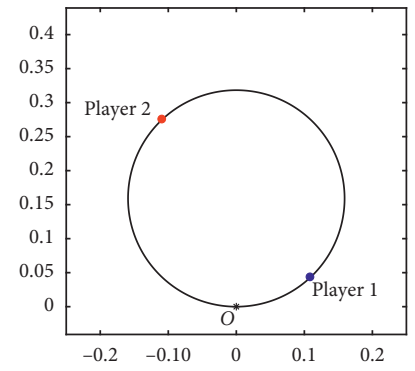

- $x_{1}=0.12$

- $x_{2}=0.62$

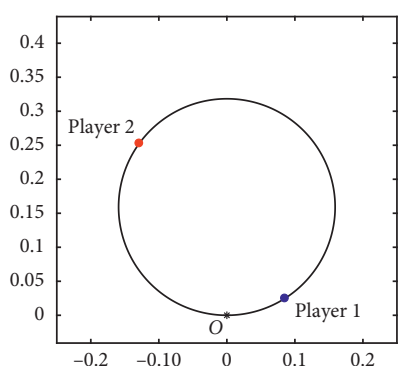

$-x_{1}=0.09$
$-x_{2}=0.65$

(d)

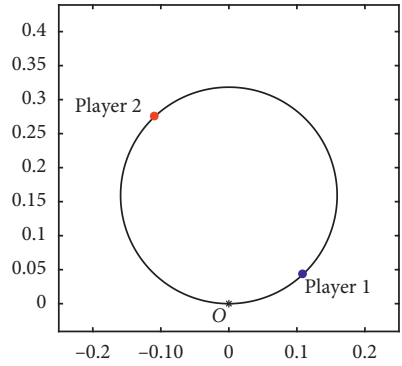

- $x_{1}=0.12$

- $x_{2}=0.62$

(g) (h)

Figure 5: Dynamic evolution of the two-player location game in the closed-loop market with initial positions $x_{1}=0.06$ and $x_{2}=0.68$.

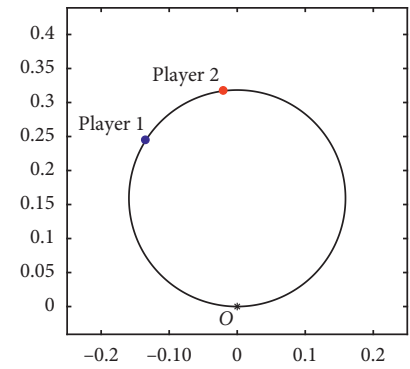

- $x_{1}=0.66$

- $x_{2}=0.52$

(a)

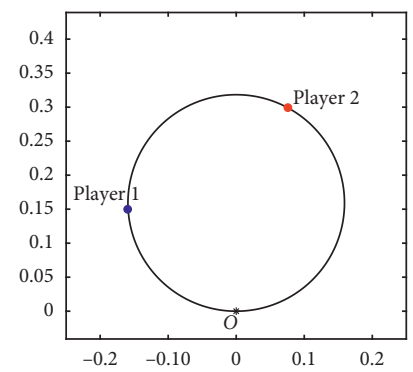

- $x_{1}=0.76$

- $x_{2}=0.42$

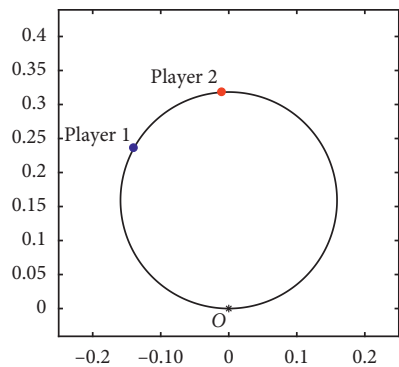

- $x_{1}=0.67$

- $x_{2}=0.51$

(b)

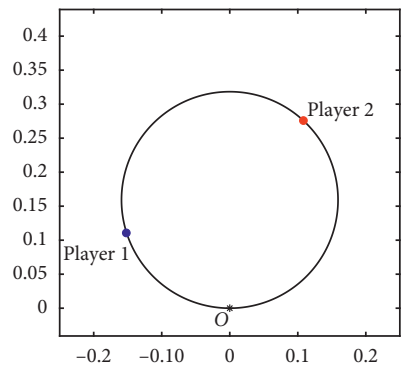

- $x_{1}=0.8$

- $x_{2}=0.38$

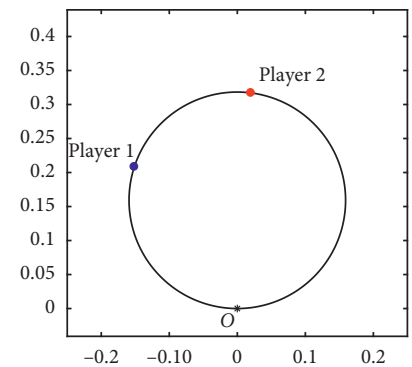

- $x_{1}=0.7$

- $x_{2}=0.48$

(c)

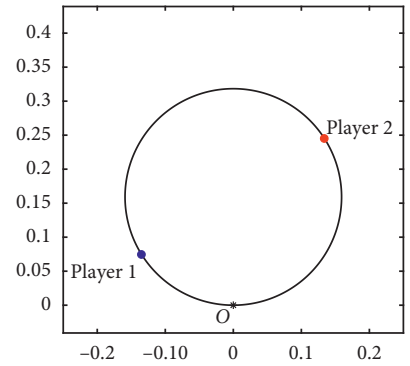

- $x_{1}=0.84$

- $x_{2}=0.34$

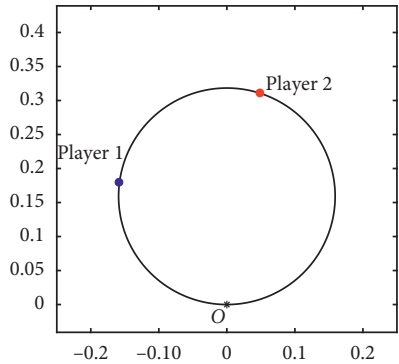

- $x_{1}=0.73$

- $x_{2}=0.45$

(d)

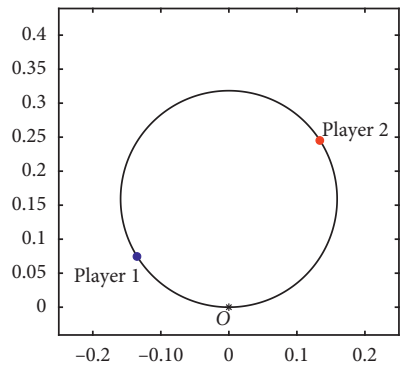

- $x_{1}=0.84$

- $x_{2}=0.34$

(h)

Figure 6: Dynamic evolution of the two-player location game in the closed-loop market with initial positions $x_{1}=0.66$ and $x_{2}=0.52$. 


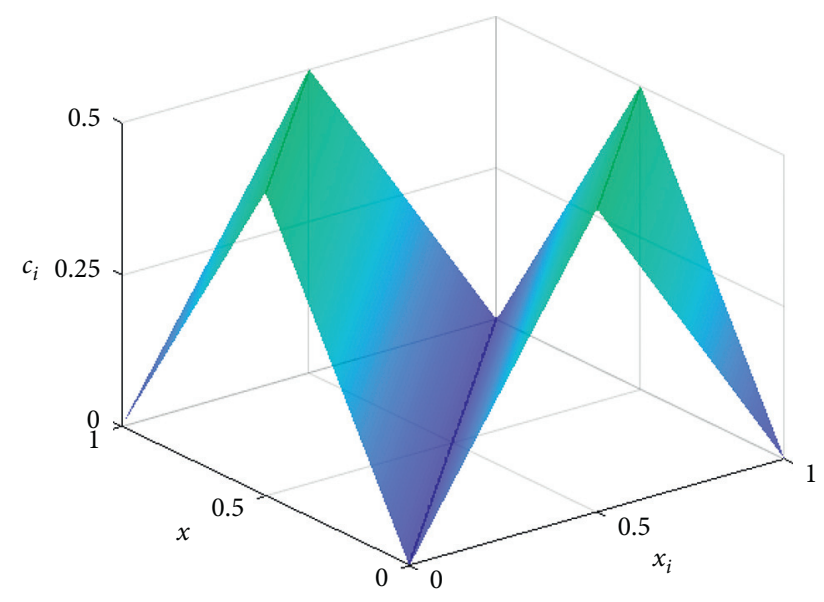

FIgUre 7: The graph of the function $c_{i}$.

\section{Conclusion}

To research the two-player location game in the closed-loop market, we consider the shape of the market as a circle. The location game with quantity competition between two players in a circular market is established. Using a two-stage approach, we solve the subgame-perfect Nash equilibria of the location game in the circular market. Moreover, we design an algorithm to simulate the dynamic evolution of the two-player location game in the closed-loop market and provide two numerical simulations to illustrate and validate the theoretical findings. The present research shows that the two players set their positions at opposite ends of a circle diameter in the optimal equilibrium state of the location game. At this point, they should adopt a differentiated quantity strategy and undifferentiated price strategy for the local markets. This paper considers the geometry of the market as a circle, which is a basic shape of the market. In fact, the geometry of the market could be very complex in the real world. Therefore, we will mainly focus on the research of multiplayer location game on complex networks in future.

Remark 6. Although the model in this paper is established under some ideal assumptions, the results have some positive reference value. In the real economic world, the whole Earth can be regarded as a circular market if observed along the Earth's latitude. There are two large countries, one in the east and the other in the west, at opposite ends of the diameter of the circular market. In theory, the competitive firms in the two countries are in an ideal equilibrium position. In fact, from the perspective of global geographical location, the firms in an equilibrium position have more location advantages than firms in other positions.

\section{Appendix}

In this appendix, we prove Lemmas 1 and 2 . Before proving them, we rewrite expression (1) of $c_{i}(x)$. Then, the relationship between $\left|x_{i}-x\right|$ and $1-\left|x_{i}-x\right|$ in (1) needs to be discussed in the following six cases. (a) $x_{i}-x>0$ and $\left|x_{i}-x\right|<1-\left|x_{i}-x\right|$, if $x_{i} \in[0,(1 / 2))$ and $x \in\left[0, x_{i}\right)$

(b) $x_{i}-x \leq 0$ and $\left|x_{i}-x\right|<1-\left|x_{i}-x\right|$, if $x_{i} \in[0,(1 / 2))$ and $x \in\left[x_{i}, x_{i}+(1 / 2)\right)$

(c) $x_{i}-x<0$ and $\left|x_{i}-x\right| \geq 1-\left|x_{i}-x\right|$, if $x_{i} \in[0,(1 / 2))$ and $x \in\left[x_{i}+(1 / 2), 1\right)$

(d) $x_{i}-x>0$ and $\left|x_{i}-x\right|>1-\left|x_{i}-x\right|$, if $x_{i} \in[(1 / 2), 1)$ and $x \in\left[0, x_{i}-(1 / 2)\right)$

(e) $x_{i}-x>0$ and $\left|x_{i}-x\right| \leq 1-\left|x_{i}-x\right|$, if $x_{i} \in[(1 / 2), 1)$ and $x \in\left[x_{i}-(1 / 2), x_{i}\right)$

(f) $x_{i}-x \leq 0$ and $\left|x_{i}-x\right|<1-\left|x_{i}-x\right|$, if $x_{i} \in[(1 / 2), 1)$ and $x \in\left[x_{i}, 1\right)$

Therefore, $c_{i}$ can be regarded as a binary function with two variables $x_{i}$ and $x$, which can be rewritten as

$$
c_{i}= \begin{cases}x_{i}-x, & \left(x_{i}, x\right) \in\left[0, \frac{1}{2}\right) \times[0, x) \cup\left[\frac{1}{2}, 1\right) \times\left[x_{i}-\frac{1}{2}, x_{i}\right), \\ x-x_{i}, & \left(x_{i}, x\right) \in\left[0, \frac{1}{2}\right) \times\left[x_{i}, x_{i}+\frac{1}{2}\right) \cup\left[\frac{1}{2}, 1\right) \times\left[x_{i}, 1\right), \\ 1-x+x_{i}, & \left(x_{i}, x\right) \in\left[0, \frac{1}{2}\right) \times\left[x_{i}+\frac{1}{2}, 1\right), \\ 1-x_{i}+x, & \left(x_{i}, x\right) \in\left[\frac{1}{2}, 1\right) \times\left[0, x_{i}-\frac{1}{2}\right) .\end{cases}
$$

Using MATLAB, the graph of the function $c_{i}$ is plotted as shown in Figure 7. Obviously, $c_{i}$ is a continuous function, but its partial derivatives do not exist at $\left|x_{i}-x\right|=0$ and $\left|x_{i}-x\right|=(1 / 2)$.

The Proof of Lemma 1. According to expression (A.1) of $c_{i}$ and by the method of subsection integration, the lemma can be proved directly. The detailed calculation process is as follows.

For $x_{i} \in[0,(1 / 2))$, we can compute

$$
\begin{aligned}
\int_{0}^{1} c_{i}(x) \mathrm{d} x= & \left.\int_{0}^{x_{i}}\left(x_{i}-x\right)\right) \mathrm{d} x+\int_{x_{i}}^{x_{i}+(1 / 2)}\left(x_{i}-x\right) \mathrm{d} x \\
& +\int_{x_{i}+(1 / 2)}^{1}\left(1-x+x_{i}\right) \mathrm{d} x \\
= & \frac{1}{2} x_{i}^{2}+\frac{1}{8}+\left(\frac{1}{8}-\frac{1}{2} x_{i}^{2}\right)=\frac{1}{4} .
\end{aligned}
$$

For $x_{i} \in[(1 / 2), 1)$, we can compute

$$
\begin{aligned}
\int_{0}^{1} c_{i}(x) \mathrm{d} x= & \int_{0}^{x_{i}-(1 / 2)}\left(1-x_{i}+x\right) \mathrm{d} x+\int_{x_{i}-(1 / 2)}^{x_{i}}\left(x_{i}-x\right) \mathrm{d} x \\
& +\int_{x_{i}}^{1}\left(x-x_{i}\right) \mathrm{d} x \\
= & \left(-\frac{1}{2} x_{i}^{2}+x_{i}-\frac{3}{8}\right)+\frac{1}{8}+\left(\frac{1}{2} x_{i}^{2}-x_{i}+\frac{1}{2}\right)=\frac{1}{4} .
\end{aligned}
$$


It follows from (A.2) and (A.3) that $\int_{0}^{1} c_{i}(x) \mathrm{d} x=(1 / 4)$. For $x_{i} \in[0,(1 / 2))$, we can compute

$$
\begin{aligned}
\int_{0}^{1} c_{i}^{2}(x) \mathrm{d} x= & \int_{0}^{x_{i}}\left(x_{i}-x\right)^{2} \mathrm{~d} x+\int_{x_{i}}^{x_{i}+(1 / 2)}\left(x_{i}-x\right)^{2} \mathrm{~d} x \\
& +\int_{x_{i}+(1 / 2)}^{1}\left(1-x+x_{i}\right)^{2} \mathrm{~d} x \\
= & \int_{0}^{x_{i}+(1 / 2)}\left(x_{i}-x\right)^{2} \mathrm{~d} x+\int_{x_{i}+(1 / 2)}^{1}\left(1-x+x_{i}\right)^{2} \mathrm{~d} x \\
= & \left(\frac{1}{3} x_{i}^{3}+\frac{1}{24}\right)+\left(\frac{1}{24}-\frac{1}{3} x_{i}^{3}\right)=\frac{1}{12} .
\end{aligned}
$$

For $x_{i} \in[(1 / 2), 1)$, we can compute

$$
\begin{aligned}
\int_{0}^{1} c_{i}^{2}(x) \mathrm{d} x= & \int_{0}^{x_{i}-(1 / 2)}\left(1-x_{i}+x\right)^{2} \mathrm{~d} x+\int_{x_{i}-(1 / 2)}^{x_{i}}\left(x_{i}-x\right)^{2} \mathrm{~d} x \\
& +\int_{x_{i}}^{1}\left(x-x_{i}\right)^{2} \mathrm{~d} x \\
= & \int_{0}^{x_{i}-(1 / 2)}\left(1-x_{i}+x\right)^{2} \mathrm{~d} x+\int_{x_{i}-(1 / 2)}^{1}\left(x_{i}-x\right)^{2} \mathrm{~d} x \\
= & \left(\frac{1}{3} x_{i}^{3}-x_{i}^{2}+x_{i}-\frac{7}{24}\right)+\left(-\frac{1}{3} x_{i}^{3}+x_{i}^{2}-x_{i}-\frac{3}{8}\right)=\frac{1}{12} .
\end{aligned}
$$

It follows from (A.4) and (A.5) that $\int_{0}^{1} c_{i}^{2}(x) \mathrm{d} x=(1 / 12)$.

\section{The Proof of Lemma 2}

(i) For $\left(x_{1}, x_{2}\right) \in[0,(1 / 2)) \times[0,(1 / 2))$, it follows from (A.1) that

$$
c_{i}(x)= \begin{cases}x_{i}-x, & x \in\left[0, x_{i}\right), \\ x-x_{i}, & x \in\left[x_{i}, x_{i}+\frac{1}{2}\right), \\ 1-x+x_{i}, & x \in\left[x_{i}+\frac{1}{2}, 1\right) .\end{cases}
$$

Consequently, for $x_{1} \leq x_{2}$, we can compute

$$
\begin{aligned}
\int_{0}^{1} c_{1}(x) c_{2}(x) \mathrm{d} x= & \int_{0}^{x_{1}}\left(x_{1}-x\right)\left(x_{2}-x\right) \mathrm{d} x+\int_{x_{1}}^{x_{2}} \\
& \cdot\left(x-x_{1}\right)\left(x_{2}-x\right) \mathrm{d} x \\
& +\int_{x_{2}}^{x_{1}+(1 / 2)}\left(x-x_{1}\right)\left(x_{2}-x\right) \mathrm{d} x+\int_{x_{1}+(1 / 2)}^{x_{2}+(1 / 2)} \\
& \cdot\left(1-x+x_{1}\right)\left(x_{2}-x\right) \mathrm{d} x \\
& +\int_{x_{2}+(1 / 2)}^{1}\left(1-x+x_{1}\right)\left(1-x+x_{2}\right) \mathrm{d} x \\
= & -\frac{2}{3}\left(x_{1}-x_{2}\right)^{3}-\frac{1}{2}\left(x_{1}-x_{2}\right)^{2}+\frac{1}{12} .
\end{aligned}
$$

Similarly, for $x_{1}>x_{2}$, the following can be calculated:

$$
\int_{0}^{1} c_{1}(x) c_{2}(x) \mathrm{d} x=\frac{2}{3}\left(x_{1}-x_{2}\right)^{3}-\frac{1}{2}\left(x_{1}-x_{2}\right)^{2}+\frac{1}{12} .
$$

Combining (A.7) and (A.8), we have

$$
\int_{0}^{1} c_{1}(x) c_{2}(x) \mathrm{d} x=\frac{2}{3}\left|x_{1}-x_{2}\right|^{3}-\frac{1}{2}\left(x_{1}-x_{2}\right)^{2}+\frac{1}{12},
$$

for $\left(x_{1}, x_{2}\right) \in[0,(1 / 2)) \times[0,(1 / 2))$.

(ii) For $\left(x_{1}, x_{2}\right) \in[0,(1 / 2)) \times[(1 / 2), 1)$, it follows from (A.1) that

$$
\begin{aligned}
& c_{1}(x)= \begin{cases}x_{1}-x, & x \in\left[0, x_{1}\right), \\
x-x_{1}, & x \in\left[x_{1}, x_{1}+\frac{1}{2}\right), \\
1-x+x_{1}, & x \in\left[x_{1}+\frac{1}{2}, 1\right),\end{cases} \\
& c_{2}(x)= \begin{cases}1-x_{2}+x, & x \in\left[0, x_{2}-\frac{1}{2}\right), \\
x_{2}-x, & x \in\left[x_{2}-\frac{1}{2}, x_{2}\right), \\
x-x_{2}, & x \in\left[x_{2}, 1\right) .\end{cases}
\end{aligned}
$$

Consequently, for $x_{1} \leq x_{2}-(1 / 2)$, the following can be computed: 


$$
\begin{aligned}
\int_{0}^{1} c_{1}(x) c_{2}(x) \mathrm{d} x= & \int_{0}^{x_{1}}\left(x_{1}-x\right)\left(1-x_{2}+x\right) \mathrm{d} x+\int_{x_{1}}^{x_{2}-(1 / 2)} \\
& \cdot\left(x-x_{1}\right)\left(1-x_{2}+x\right) \mathrm{d} x \\
& +\int_{x_{2}-(1 / 2)}^{x_{1}+(1 / 2)}\left(x-x_{1}\right)\left(x_{2}-x\right) \mathrm{d} x+\int_{x_{1}+(1 / 2)}^{x_{2}} \\
& \cdot\left(1-x+x_{1}\right)\left(x_{2}-x\right) \mathrm{d} x \\
& +\int_{x_{2}}^{1}\left(1-x+x_{1}\right)\left(x-x_{2}\right) \mathrm{d} x \\
= & \frac{2}{3}\left(x_{1}-x_{2}+\frac{1}{2}\right)^{3}+\frac{1}{2}\left(x_{1}-x_{2}+\frac{1}{2}\right)^{2}+\frac{1}{24} .
\end{aligned}
$$

Similarly, for $x_{1}>x_{2}-(1 / 2)$, we can compute

$\int_{0}^{1} c_{1}(x) c_{2}(x) \mathrm{d} x=-\frac{2}{3}\left(x_{1}-x_{2}+\frac{1}{2}\right)^{3}+\frac{1}{2}\left(x_{1}-x_{2}+\frac{1}{2}\right)^{2}+\frac{1}{24}$.

It follows from (A.11) and (A.12) that

$$
\int_{0}^{1} c_{1}(x) c_{2}(x) \mathrm{d} x=-\frac{2}{3}\left|x_{1}-x_{2}+\frac{1}{2}\right|^{3}+\frac{1}{2}\left(x_{1}-x_{2}+\frac{1}{2}\right)^{2}+\frac{1}{24},
$$

$$
\text { if }\left(x_{1}, x_{2}\right) \in[0,(1 / 2)) \times[(1 / 2), 1) \text {. }
$$

(iii) For $\left(x_{1}, x_{2}\right) \in[(1 / 2), 1) \times[0,(1 / 2))$, as in (ii), we can compute

$$
\int_{0}^{1} c_{1}(x) c_{2}(x) \mathrm{d} x=-\frac{2}{3}\left|x_{2}-x_{1}+\frac{1}{2}\right|^{3}+\frac{1}{2}\left(x_{2}-x_{1}+\frac{1}{2}\right)^{2}+\frac{1}{24} .
$$

(iv) For $\left(x_{1}, x_{2}\right) \in[(1 / 2), 1) \times[(1 / 2), 1)$, according to (A.1), $c_{i}(x)$ can be reduced to

$$
c_{i}(x)= \begin{cases}1-x_{i}+x, & x \in\left[0, x_{i}-\frac{1}{2}\right), \\ x_{i}-x, & x \in\left[x_{i}-\frac{1}{2}, x_{i}\right), \\ x-x_{i}, & x \in\left[x_{i}, 1\right) .\end{cases}
$$

Consequently, for $x_{1} \leq x_{2}$, we can compute

$$
\begin{aligned}
\int_{0}^{1} c_{1}(x) c_{2}(x) \mathrm{d} x= & \int_{0}^{x_{1}-(1 / 2)} c_{1}(x) c_{2}(x) \mathrm{d} x+\int_{x_{1}-(1 / 2)}^{x_{2}-(1 / 2)} c_{1}(x) c_{2} \\
& \cdot(x) \mathrm{d} x+\int_{x_{2}-(1 / 2)}^{x_{1}} c_{1}(x) c_{2}(x) \mathrm{d} x+\int_{x_{1}}^{x_{2}} c_{1} \\
& \cdot(x) c_{2}(x) \mathrm{d} x+\int_{x_{2}}^{1} c_{1}(x) c_{2}(x) \mathrm{d} x \\
= & -\frac{2}{3}\left(x_{1}-x_{2}\right)^{3}-\frac{1}{2}\left(x_{1}-x_{2}\right)^{2}+\frac{1}{24} .
\end{aligned}
$$

Similarly, for $x_{1}>x_{2}$, we can computethe following:

$$
\int_{0}^{1} c_{1}(x) c_{2}(x) \mathrm{d} x=\frac{2}{3}\left(x_{1}-x_{2}\right)^{3}-\frac{1}{2}\left(x_{1}-x_{2}\right)^{2}+\frac{1}{24} \text {. }
$$

It follows from (A.16) and (A.17) that

$$
\int_{0}^{1} c_{1}(x) c_{2}(x) \mathrm{d} x=\frac{2}{3}\left|x_{1}-x_{2}\right|^{3}-\frac{1}{2}\left(x_{1}-x_{2}\right)^{2}+\frac{1}{24},
$$

for $\left(x_{1}, x_{2}\right) \in[(1 / 2), 1) \times[(1 / 2), 1)$.

Specifically, for $x_{1}=x_{2}$, it follows from (A.13) and (A.18) that

$$
\int_{0}^{1} c_{1}(x) c_{2}(x) \mathrm{d} x=\frac{1}{12} .
$$

For $\left|x_{1}-x_{2}\right|=(1 / 2)$, according to (A.13) and (A.14), we can obtain

$$
\int_{0}^{1} c_{1}(x) c_{2}(x) \mathrm{d} x=\frac{1}{24}
$$

\section{Data Availability}

The data used to support the findings of this study are available from the corresponding author upon request.

\section{Conflicts of Interest}

The authors declare that there are no conflicts of interest regarding the publication of this paper.

\section{Acknowledgments}

This work was supported in part by the National Natural Science Foundation of China under Grant 61773004, in part by the Natural Science Foundation Project of Chongqing under Grant cstc2018jcyjAX0606, and in part by the Science and Technology Research Program of Chongqing Municipal Education Commission under Grant KJQN201900701. 


\section{References}

[1] J. von Neumann, "Zur theorie der gesellschaftsspiele," Mathematische Annalen, vol. 100, no. 1, pp. 295-320, 1928.

[2] T. Ichiishi, A. Neyman, Y. Tauman, and K. Shell, Game Theory and Applications, Academic Press, Cambridge, MA, USA, 1990.

[3] S. Lasaulce and H. Tembine, Game Theory and Learning for Wireless Networks: Fundamentals and Applications, Academic Press, Cambridge, MA, USA, 2011.

[4] P. D. Giovanni and G. Zaccour, "A two-period game of a closed-loop supply chain," European Journal of Operational Research, vol. 232, pp. 22-40, 2014.

[5] S. Goyal, H. Heidari, and M. Kearns, "Competitive contagion in networks," Games and Economic Behavior, vol. 113, pp. 58-79, 2019.

[6] E. Talamàs, "Price dispersion in stationary networked markets," Games and Economic Behavior, vol. 115, pp. 247-264, 2019.

[7] W.-C. Guo and F.-C. Lai, "Spatial cournot competition in two intersecting circular markets," The Annals of Regional Science, vol. 64, no. 1, pp. 37-56, 2020.

[8] H. Hotelling, "Stability in competition," The Economic Journal, vol. 39, no. 153, pp. 41-57, 1929.

[9] C. d'Aspremont, J. J. Gabszewicz, and J.-F. Thisse, "Computation of multi-facility location Nash equilibria on a network under quantity competition," Econometrica, vol. 47, no. 4, pp. 1145-1150, 1979.

[10] B. Gupta, D. Pal, and J. Sarkar, "Spatial Cournot competition and agglomeration in a model of location choice," Regional Science and Urban Economics, vol. 27, no. 3, pp. 261-282, 1997.

[11] E. Gal-or, "Hotelling's spatial competition as a model of sales," Economics Letters, vol. 9, no. 1, pp. 1-6, 1982.

[12] D. Graitson, "Spatial competition a la Hotelling: a selective survey," The Journal of Industrial Economics, vol. 31, no. 1/2, pp. 11-25, 1982.

[13] N. Economides, "Minimal and maximal product differentiation in Hotelling's duopoly," Economics Letters, vol. 21, no. 1, pp. 67-71, 1986.

[14] S. P. Anderson, J. K. Goeree, and R. Ramer, "Location, location, location," Journal of Economic Theory, vol. 77, no. 1, pp. 102-127, 1997.

[15] S. Brenner, "Hotelling games with three, four, and more players," Journal of Regional Science, vol. 45, no. 4, pp. 851-864, 2005.

[16] L. Lambertini and R. Orsini, "On Hotelling's "stability in competition" with network externalities and switching costs," Papers in Regional Science, vol. 92, no. 4, pp. 873-883, 2013.

[17] W.-C. Guo, F.-C. Lai, and D.-Z. Zeng, "A Hotelling model with production," Mathematical Social Sciences, vol. 73, pp. 40-49, 2015.

[18] J. Hinloopen and S. Martin, "Costly location in Hotelling duopoly," Research in Economics, vol. 71, no. 1, pp. 118-128, 2017.

[19] J. Chen and B. Chen, "When should the offline retailer implement price matching?" European Journal of Operational Research, vol. 277, no. 3, pp. 996-1009, 2019.

[20] M. A. de Frutos, H. Hamoudi, and X. Jarque, "Equilibrium existence in the circle model with linear quadratic transport cost," Regional Science and Urban Economics, vol. 29, no. 5, pp. 605-615, 1999.
[21] M. Peitz, "The circular road revisited: uniqueness and supermodularity," Research in Economics, vol. 53, no. 4, pp. 405-420, 1999.

[22] B. Gupta, F.-C. Lai, D. Pal, J. Sarkar, and C.-M. Yu, "Where to locate in a circular city?" International Journal of Industrial Organization, vol. 22, no. 6, pp. 759-782, 2004.

[23] T. Matsumura and D. Shimizu, "Cournot and Bertrand in shipping models with circular markets," Papers in Regional Science, vol. 85, no. 4, pp. 585-598, 2006.

[24] D. Pal and J. Sarkar, "Spatial Cournot competition among multi-plant firms in a circular city," Southern Economic Journal, vol. 73, no. 1, pp. 246-258, 2006.

[25] Q. Gong, Q. Liu, and Y. Zhang, "Optimal product differentiation in a circular model," Journal of Economics, vol. 119, no. 3, pp. 219-252, 2016.

[26] C.-H. Sun, J.-F. Tsai, and F.-C. Lai, "Spatial Cournot competition in a circular city with more than two dispatches," The Japanese Economic Review, vol. 68, no. 4, pp. 413-442, 2017.

[27] A. Cournot, Recherches sur les Principes Mathématiques de la Théoris des Richesses, Hachette, Paris, France, 1838. 